\title{
Spiroconjugation-enhanced intermolecular charge transport
}

\author{
Chung-Chih Wu, ${ }^{\text {a) }}$ Wei-Guang Liu, Wen-Yi Hung, Tsung-Li Liu, \\ Yu-Ting Lin, and Hao-Wu Lin \\ Department of Electrical Engineering, Graduate Institute of Electro-optical Engineering, and Graduate \\ Institute of Electronics Engineering, National Taiwan University, Taipei, Taiwan 106, \\ Republic of China
}

Ken-Tsung Wong, Yuh-Yih Chien, Ruei-Tang Chen, Tsung-Hsi Hung, Teng-Chih Chao, and You-Ming Chen

Department of Chemistry, National Taiwan University, Taipei, Taiwan, 106, Republic of China

(Received 29 April 2005; accepted 21 June 2005; published online 26 July 2005)

\begin{abstract}
We report charge-transport and related physical properties of terfluorenes with various C9 substitutions. Results show that by introducing slight spiroconjugation between a core chromophore and conjugated substitutions through spiro-linking, the bulky substitution not only serves as a spatial hindrance to enhance morphological stability and emission efficiency in thin films, but also bridges and enhances intermolecular charge transport under certain situations. Interestingly, such characteristics are acquired without altering major electronic properties of core chromophores, rendering it a flexible molecular scheme for tuning characteristics of functional molecules to meet various demands of different applications. (C) 2005 American Institute of Physics.

[DOI: 10.1063/1.2001140]
\end{abstract}

Intermolecular charge transport is a central concern of both fundamental and practical nature for functional molecules for optoelectronic applications. ${ }^{1-6}$ Very often in designing functional molecules, to acquire certain functionalities, such as particular molecular conformations, intermolecular spatial hindrance, and desired morphological characteristics in condensed phases, yet without altering major electronic or optical properties of a core chromophore, bulky and inert peripheral substitution may be introduced. ${ }^{4-6}$ The issue long associated with such a general strategy, however, is that bulky and inert substitution would impede intermolecular charge transfer, degrading carrier-transport properties essential for various applications. In this letter, we investigate the influences of peripheral substitutions on carrier transport of terfluorenes, which have been recently reported to exhibit unusual nondispersive bipolar carrier transport and high carrier mobilities in the amorphous state. ${ }^{7}$ Convenient substitution on $\mathrm{C} 9$ of fluorene moieties provides terfluorenes with structural variety, which in turn gives one valuable opportunities to systematically probe influences of molecular structures, particularly peripheral substitution, on intermolecular charge transport (both hole transport and electron transport). Results here show that, without significantly altering major electronic properties of the core chromophore, some type of substitution, in addition to their benefits to morphological and photophysical properties, may indeed enhance intermolecular charge transport.

Terfluorenes with three different types of C9 substitutions, as shown in Fig. 1, were prepared and characterized according to protocols reported previously. ${ }^{8}$ They include the one with alkyl substituents (E3), one with diaryl substituents (T3) and one with spiro-linked conjugated substituents (B3). Table I summarizes various physical properties of these terfluorenes. In spite of large variation of C9 substitution, all terfluorenes exhibit very similar photophysical properties:

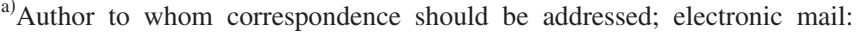
chungwu@cc.ee.ntu.edu.tw nearly the same lowest-energy absorption bands around $350 \mathrm{~nm}$, and similar photoluminescence (PL) spectra in either solutions (with PL peaks around 392-395 and 411-415 nm) or vacuum-deposited thin films (with PL peaks around 403-405 and $427 \mathrm{~nm}$ ). These transition properties agree with characteristics of the lowest $\pi-\pi^{*}$ transition of the central terfluorene chromophore, and appear consistent with the usual viewpoint that the tetrahedral C9 carbon (i.e., the spiro center) serves as an effective insulating spacer blocking interaction between the central chromophore and the $\mathrm{C} 9$ substitution. ${ }^{9,10}$ On the other hand, the bulky and rigid C9 substitution of T3 and B3 apparently is highly beneficial to emission efficiency in the solid state by suppressing quenching due to interchromophore interaction, and to morphological stability of thin films by providing steric spatial hindrance. In going from solutions to thin films, PL quantum yield of E3 drops substantially from $82 \%$ to $50 \%$, while those of T3 and B3 only drop a few percent. Without the bulky substitution, E3 exhibits a glass-transition temperature $\left(104^{\circ} \mathrm{C}\right)$ substantially lower than those of B3 $\left(237^{\circ} \mathrm{C}\right)$ and T3 $\left(204{ }^{\circ} \mathrm{C}\right)$.

Carrier-transport properties of terfluorenes in vacuumdeposited films were characterized by the time-of-flight (TOF) transient photocurrent technique. ${ }^{2,7} \mathrm{E} 3, \mathrm{~T} 3$, and B3 all exhibit nondispersive bipolar carrier transport, and roomtemperature hole mobilities $\mu_{h}$ 's and electron mobilities $\mu_{e}$ 's for three compounds as a function of the square root of the applied electric field $\left(E^{1 / 2}\right)$ are compared in Figs. 2(a) and 2(b), respectively. Influences of different $C 9$ substitutions on carrier transport depend on the type of carriers. For hole transport, $\mu_{h}$ 's are in the order of B3>E3 $>\mathrm{T} 3$, and $\mu_{h}$ of

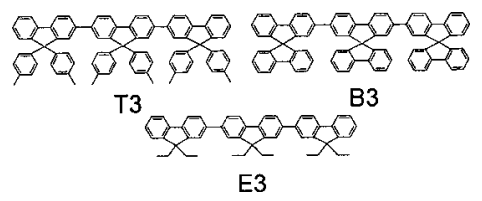

FIG. 1. Structures of the three terfluorene derivatives, E3, T3, and B3. 
TABLE I. Summary of physical properties of terfluorenes.

\begin{tabular}{|c|c|c|c|c|c|c|c|c|c|}
\hline & \multirow[b]{2}{*}{$\begin{array}{c}T_{g}{ }^{\mathrm{a}} \\
\left({ }^{\circ} \mathrm{C}\right)\end{array}$} & \multicolumn{2}{|c|}{$\lambda_{\max , \text { abs }}(\mathrm{nm})^{\mathrm{b}}$} & \multicolumn{2}{|c|}{$\lambda_{\max , \mathrm{PL}}(\mathrm{nm})^{\mathrm{c}}$} & \multicolumn{2}{|c|}{$Q Y^{\mathrm{d}}$} & \multicolumn{2}{|c|}{$\lambda(\mathrm{meV})^{\mathrm{e}}$} \\
\hline & & Solution & Film & Solution & Film & Solution & Film & Hole & Electron \\
\hline E3 & 104 & 351 & 346 & 395,415 & 403,427 & 0.82 & 0.50 & 214 & 290 \\
\hline T3 & 204 & 355 & 352 & 393,414 & 405,427 & 0.85 & 0.80 & 219 & 285 \\
\hline B3 & 237 & 352 & 352 & 392,411 & 405,427 & 0.97 & 0.90 & 182 & 279 \\
\hline
\end{tabular}

${ }^{a}$ Glass transition temperatures recorded by differential scanning calorimetry.

${ }^{b}$ First absorption peaks in solutions $\left(1 \times 10^{-5} \mathrm{M}\right.$ in $\left.\mathrm{CHCl}_{3}\right)$ and in films.

${ }^{\mathrm{c}} \mathrm{PL}$ peaks in solutions $\left(1 \times 10^{-4} \mathrm{M}\right.$ in $\left.\mathrm{CHCl}_{3}\right)$ and in films.

${ }^{\mathrm{d}} \mathrm{PL}$ quantum yields in solutions and thin films, determined by a calibrated integrating sphere system.

${ }^{\mathrm{e}}$ Reorganiztion energies for hole and electron transport.

B3 is about twice that of E3, and one order higher than that of T3. For electron transport, $\mu_{e}$ 's are in the order of E3 $>\mathrm{B} 3 \approx \mathrm{T} 3$, with the electron mobility of $\mathrm{E} 3$ being over three times higher than those of $\mathrm{B} 3$ and $\mathrm{T} 3$.

Observation of efficient bipolar transport in E3 indicates that it is intrinsic property of terfluorenes, precluding such unusual carrier-transport properties from being necessarily associated with the attachment of rigid and bulky aryl or conjugated C9 substituents. ${ }^{7}$ Different influences of the substitutions on hole and electron transport, however, are not readily explained by assuming an inert role of the $\mathrm{C} 9$ substitution in carrier transport. Measurements of mass densities of vacuum-deposited terfluorene films give densities of $1.16 \mathrm{~g} / \mathrm{cm}^{3}$ for $\mathrm{E} 3$ and $\sim 1 \mathrm{~g} / \mathrm{cm}^{3}$ for $\mathrm{T} 3$ and $\mathrm{B} 3$, corresponding to average volume/molecule of 950 and $1728 \AA^{3}$ and average distance between terfluorene backbones of 6.2 and $\sim 8.3 \AA$ (assuming the terfluorene backbone length of $\sim 25 \AA$ A). ${ }^{11}$ Supposing carriers transport through hopping between core chromophores, one would expect E3 to perform best in both hole transport and electron transport with its smallest inter-chromophore spacing and expect $\mathrm{B} 3$ and $\mathrm{T} 3$ to behave similar due to similar bulkiness of the $\mathrm{C} 9$ aryl substitution. This appears to be the case for electron transport, but not for hole transport. Although T3 does have a lower $\mu_{h}$ than E3 as expected, B3 has over one order higher $\mu_{h}$ than T3 despite similarly bulky C9 substitution and has even higher $\mu_{h}$ than E3.

At the microscopic level, intermolecular charge transport can be regarded as electron transfer (ET) between neighboring charged and neutral molecules. ${ }^{12,13}$ According to Marcus ET theory, ${ }^{12,13}$ the rate for charge hopping $\left(k_{\mathrm{ET}}\right)$ has the dependence of

$$
k_{\mathrm{ET}} \propto t^{2} \exp \left(-\frac{\lambda}{4 k_{B} T}\right) .
$$

Here $\lambda$ is internal reorganization energy which corresponds to the sum of geometry relaxation energies upon going from
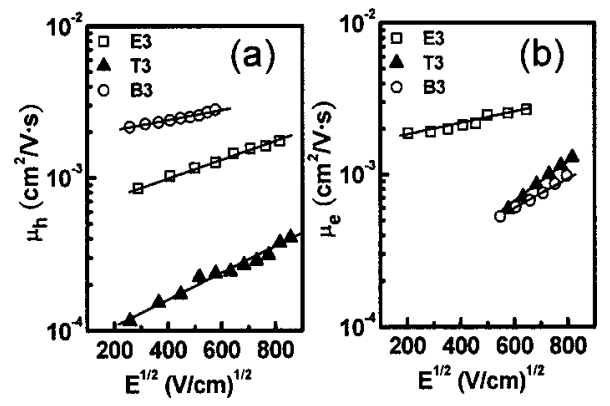

FIG. 2. Room-temperature carrier mobilities of terfluorenes: (a) Hole mo-

bility $\mu_{h}$ vs $E^{1 / 2}$, (b) electron mobility $\mu_{e}$ vs $E^{1 / 2}$ between the two moieties (terfluorene and biphenyl), elec-
Downloaded 17 Feb 2009 to 140.112 .113 .225 . Redistribution subject to AlP license or copyright; see http://apl.aip.org/apl/copyright.jsp the neutral-state geometry to the charged-state geometry and vice versa. The term $t$ represents the strength of intermolecular electronic coupling (transfer integral) between adjacent molecules. Reorganization energies of three terfluorenes can be estimated with quantum chemical calculation, ${ }^{14,15}$ and are listed in Table I. Reorganization energy related to hole transport of B3 is $\sim 30-40 \mathrm{meV}$ lower than those of E3 and T3 presumbly due to more rigid structures and larger conjugation (see the following paragraph) in B3. Such difference can only partly account for the higher $\mu_{h}$ of B3 relative to those of E3 and T3. Satisfactory explanation of enhanced $\mu_{h}$ of B3 requires its intermolecular electronic coupling $(t)$ for holes to be substantially stronger than those of E3 and T3. While quantitative estimation of $t$ is not possible without knowing exact spatial arrangements of molecules in the present disordered systems, the examination of molecular orbitals and thin-film morphologies of terfluorenes provides some hints as to how this may happen.

Figure 3 shows frontier orbitals [highest occupied molecular orbitals (HOMOs) and lowest unoccupied molecular orbitals (LUMOs)] on optimized molecular structures of terfluorenes. ${ }^{16}$ Frontier MOs of all three terfluorenes have similar appearance and are mainly localized on the terfluorene backbones. Yet HOMO of B3, unlike other MOs shown, exhibits a relatively significant distribution on C9 substitutions, particularly on the central one. Such a behavior is surprising at a first glance, considering that the biphenyl and terfluorene moieties are bonded in an orthogonal relationship through a $\mathrm{SP}^{3}$ carbon, which in principle should give minimal interaction. Early studies back in 1960's, however, indicate that it is a natural consequence of "sprioconjugation," an interaction that allows for electronic coupling between two mutually perpendicular $\pi$ subsystems joined by a spiro atom. ${ }^{17,18}$ Such interaction occurs if the fragment MOs of the original $\pi$ subsystems near the spiro center have the correct symmetry (i.e., both fragment MOs are antisymmetric to planes of both $\pi$ subsystems). Spiroconjugation leads to MO mixing of original subsystems and produces MOs spanning the entire system. Inspecting symmetries of frontier MOs of terfluorene and various C9 substitutions, only HOMOs of terfluorene and biphenyl in B3 have the required symmetry, explaining extension of HOMO to the biphenyl substitution. The weighting of MO mixing and energy splitting through spiroconjugation depends on the similarity in $\mathrm{MO}$ energies of original subsystems. For the case of B3, the distribution of HOMO on biphenyls is up to a few percent. Owing to still rather small HOMO distribution on biphenyl, extreme localization of LUMO on terfluorene, and orthogonal relationship between the two moieties (terfluorene and biphenyl), electo AlP license or copyright; see http://apl.aip.org/apl/copyright.jsp 

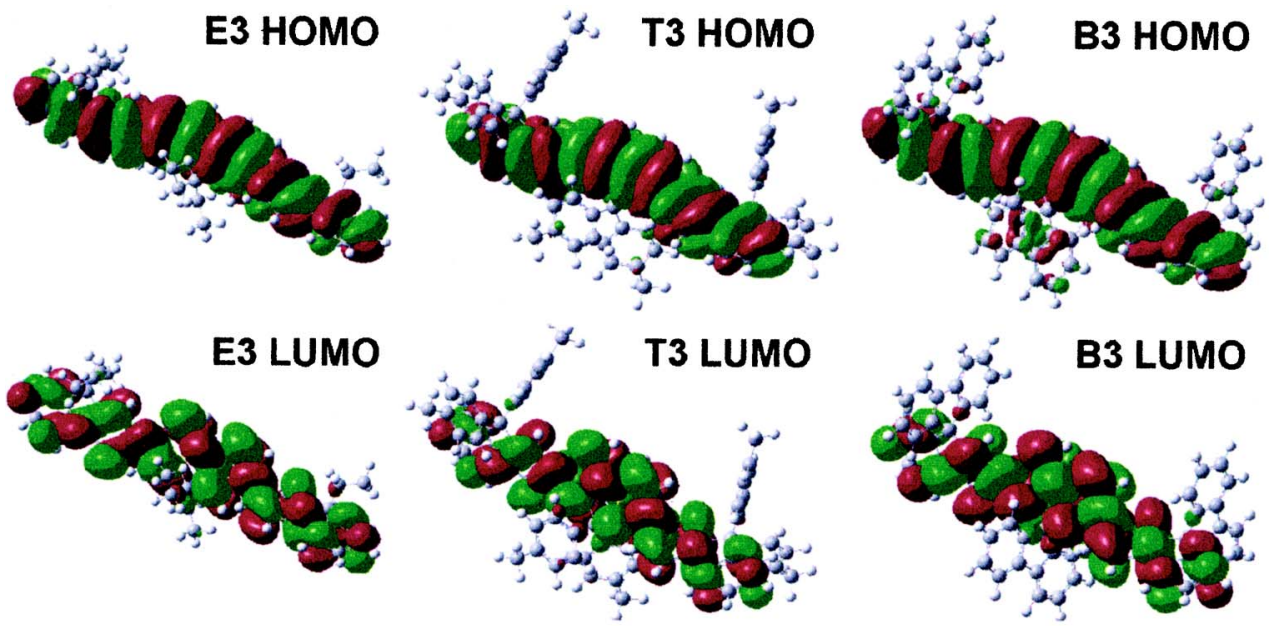

FIG. 3. (Color) Frontier molecular orbitals (HOMOs and LUMOs) of terfluorenes on optimized molecular structures.

tronic transitions between frontier MOs of B3 can be considered involving the terfluorene moiety only. Thus B3 exhibits photophysical properties similar to the other two derivatives, even though HOMO spiroconjugation occurs.

Previous characterization of vacuum-deposited terfluorene films by spectroscopic ellipsometry reveals their anisotropic molecular stacking, ${ }^{19}$ i.e., with molecular axes lying roughly parallel to the substrate surface, even though films are amorphous. Under such situations, molecules are naturally arranged with $\mathrm{C} 9$ substituents interpenetrated between backbones, as illustrated in Fig. 4. Thus, although the spacing between backbones may be large, the spacing between one backbone and the substituent of another neighboring backbone or the spacing between substituents of two neighboring backbones could be close. In such a format, for carrier transport roughly perpendicular to terfluorene backbones as in the TOF measurement, $\mathrm{C} 9$ substitutions function either as insulating spatial hindrance (e.g., carrier transport in E3 and $\mathrm{T} 3$, and electron transport in B3) or as active bridging (hole transport in B3), depending on whether corresponding MOs extend to the substituents. In films of B3, even though the average spacing between terfluorene backbones increases (vs E3), the effective intermolecular electronic coupling for hole transport may not correspondingly decrease as in carrier transport of T3 or as in its own electron transport. In comparing hole-transport properties of E3, T3, and B3, one concludes that the effect of HOMO extension due to spiroconjugation not only compensates for negative influences of larger inter-chromophore spacing, but even further enhances intermolecular charge transport.

In summary, we have studied charge-transport and related physical properties of terfluorenes with various C9 sub-

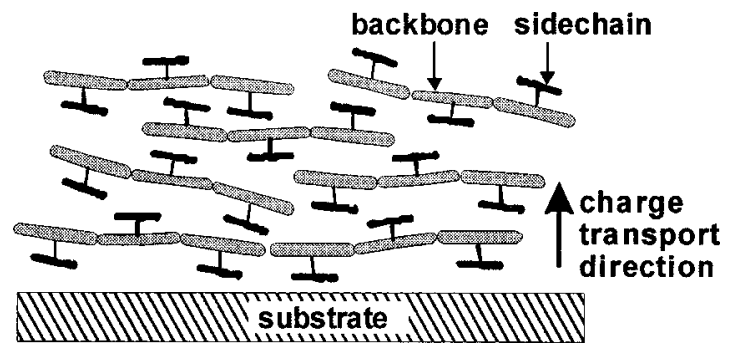

FIG. 4. Schematic illustration of morphologies of terfluorene thin films. Downloaded 17 Feb 2009 to 140.112.113.225. Redistribution subject stitutions. Results show that without altering major electronic properties of the core chromophore, introducing spiroconjugated substitution not only provides a spatial hindrance to enhance morphological stability and emission efficiency in solid states, but also bridges and enhances intermolecular charge transport. Such a molecular scheme may be useful for tuning characteristics of functional molecules to meet various demands of different applications.

The authors gratefully acknowledge the financial support from the National Science Council and Evervictory Acutech Corporation of the Republic of China.

${ }^{1}$ C. W. Tang and S. A. VanSlyke, Appl. Phys. Lett. 51, 913 (1987).

${ }^{2}$ P. M. Borsenberger and D. S. Weiss, Organic Photoreceptors for Imaging Systems (Dekker, New York, 1993).

${ }^{3}$ P. M. Lundquist, R. Wortmann, C. Geletneky, R. J. Twieg, M. Jurich, V. Y. Lee, C. R. Moylan, and D. M. Burland, Science 274, 1182 (1996).

${ }^{4}$ K. Naito and A. Miura, J. Phys. Chem. 97, 6240 (1993).

${ }^{5}$ Y. Shirota, J. Mater. Chem. 10, 1 (2000).

${ }^{6}$ P. Strohriegl and J. V. Grazulevicius, Adv. Mater. (Weinheim, Ger.) 14, 1439 (2002).

${ }^{7}$ C.-C. Wu, T.-L. Liu, W.-Y. Hung, Y.-T. Lin, K.-T. Wong, R.-T. Chen, Y.-M. Chen, and Y.-Y. Chien, J. Am. Chem. Soc. 125, 3710 (2003).

${ }^{8}$ W. K.-T. Wong, Y.-Y. Chien, R.-T. Chen, C.-F. Wang, Y.-T. Lin, H.-H. Chiang, P.-Y. Hsieh, C.-C. Wu, C.-H. Chou, Y. O. Su, G.-H. Lee, and S.-M. Peng, J. Am. Chem. Soc. 124, 11576 (2002).

${ }^{9}$ K.-H. Weinfurtner, F. Weissörtel, G. Harmgarth, and J. Salbeck, Proc. SPIE 3476, 40 (1998).

${ }^{10}$ J. Salbeck, N. Yu, J. Bauer, F. Weissörtel, and H. Bestgen, Synth. Met. 91, 209 (1997).

${ }^{11}$ The amount (mass) of materials deposited was monitored with the quartz crystal microbalance and the thicknesses of films were confirmed by profilometry or ellipsometry, from which densities of materials in films were determined.

${ }^{12}$ J. Cornil, D. Beljonne, J.-P. Calbert, and J.-L. Brédas, Adv. Mater. (Weinheim, Ger.) 13, 1053 (2001).

${ }^{13}$ R. A. Marcus, Rev. Mod. Phys. 65, 599 (1993).

${ }^{14}$ J. C. Sancho-García, G. Horowitz, and J. L. Brédas, J. Chem. Phys. 119, 12563 (2003).

${ }^{15}$ In calculating reorganization energies with the Gaussian 03 program suite, the geometries of the neutral, cation, and anion species of terfluorene derivatives were optimized at the DFT level by using the B3LYP functionals and the $6-31 \mathrm{G}$ basis set.

${ }^{16} \mathrm{HOMOs}$ and LUMOs of terfluorenes were calculated using the HartreeFock method with 6-31G basis at the optimized conformation.

${ }^{17}$ H. E. Simmons and T. Fukunaga, J. Am. Chem. Soc. 89, 5208 (1967).

${ }^{18}$ P. Maslak and A. Chopra, J. Am. Chem. Soc. 115, 9331 (1993).

${ }^{19}$ H.-W. Lin, C.-L. Lin, H.-H. Chang, Y.-T. Lin, C.-C. Wu, Y.-M. Chen, R.-T. Chen, Y.-Y. Chien, and K.-T. Wong, J. Appl. Phys. 95, 881 (2004). to AIP license or copyright; see http://apl.aip.org/apl/copyright.jsp 